\title{
A High-Speed, Low Power Consumption Positive Edge Triggered D Flip-Flop for High Speed Phase Frequency Detector in $180 \mathrm{~nm}$ CMOS Technology
}

\author{
Prof. R .H. Talwekar, Prof. (Dr.) S.S Limaye \\ Deptt. Of Electronics and Telecommunication, DIMAT, Raipur. \\ Deptt. Of Electronics and Telecommunication, JIT, Nagpur. \\ ur_talwekar@yahoo.com, shyam_limaye@hotmail.com
}

\begin{abstract}
A high speed low power consumption positive edge triggered Delayed (D) flip-flop was designed for increasing the speed of counter in Phase locked loop, using $180 \mathrm{~nm}$ CMOS technology. The designed counter has been used in the divider chip of the phase locked loop. A divide counter is required in the feedback loop to increase the VCO frequency above the input reference frequency. The proposed circuit is faster than conventional circuit as it has fast reset operation. The circuit consumes less power as it prevents short circuit power consumption. The circuit operates at $1.8 \mathrm{~V}$ power supply. This work has been used in the design. of $2.4 \mathrm{GHz}$ CMOS PLL targeting OFDM application. The CMOS based fast D-ff circuit has designed and simulated by Virtuoso tool of CADENCE spectre
\end{abstract}

\section{KEYWORDS}

Phase locked loop (PLL), Delayed flip-flop (D-ff), Phase frequency detector (PFD),True signal phase clock (TSPC), Voltage controlled oscillator (VCO), Charge pump (CP), Divider (Div), Low pass filter (LPF).

\section{INTRODUCTION}

D flip-flop is an important part of the modern digital circuits. Phase locked loop with an excellent performance is widely studied in recent years. Frequency divider and PFD are indispensable modules of PLL, which uses D flip-flop as an integral part. Edge Triggered D flip flops are often implemented in integrated high speed operations using dynamic logic. This means that the digital output is stored on parasitic device capacitance while the device is not transitioning. This design of dynamic flip flops also enables simple resetting since the reset operation can be performed by simply discharging one or more internal nodes. The conventional D flip-flop which uses E-TSPC logic has higher operating frequencies but it features static power dissipation. However this causes small increase in power dissipation, since at the frequencies of interest dynamic power consumption is dominant. In the proposed circuit dynamic power consumption was reduced by lowering internal switching and speed is increased by shortening input to output path. The paper is organized as follows. Description of phase locked loop is done in section 2. Circuit diagram of conventional and proposed D-ff is presented in section 3 and section 4 respectively. In section 5, simulation results and graphs are presented. Section 6 will draw a conclusion.

DOI : $10.5121 /$ vlsic.2012.3513 
International Journal of VLSI design \& Communication Systems (VLSICS) Vol.3, No.5, October 2012

\section{PHASE LOCKED LOOP (PLL)}

Phase locked loop is mostly used in wireless communication and data recovery circuits. At present for above mentioned application a low voltage low area and high performance integrated circuits are used which complicates the implementation of such type of integrated circuit.

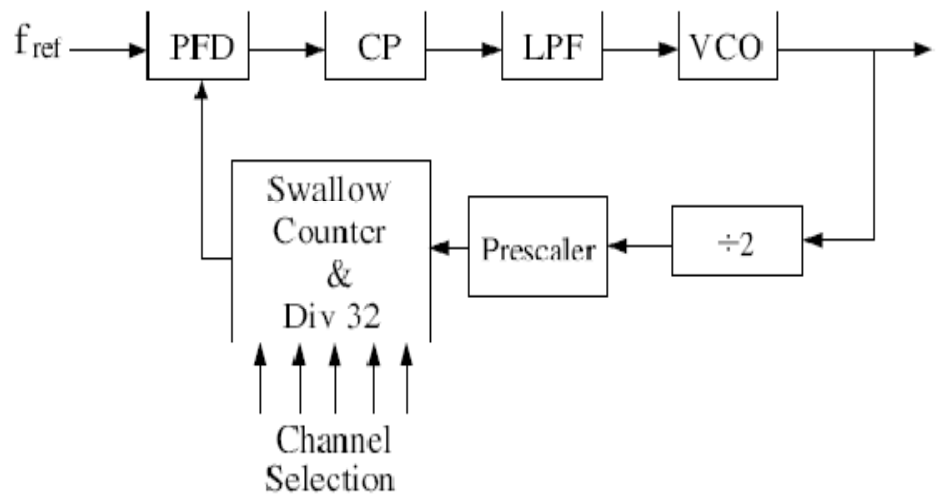

Fig. 2.1 Block diagram of PLL

A PLL is a control system that generates a signal that has a fixed relation to the phase of a "reference" signal. A phase-locked loop circuit responds to both the frequency and the phase of the input signals, automatically raising or lowering the frequency of a controlled oscillator until it is matched to the reference in both frequency and phase. Phase locked loops are built of a detector, charge pump, low pass filter, voltage-controlled oscillator (VCO) and frequency divider placed in a negative feedback closed-loop configuration. A phase detector compares two input signals and produces an error signal which is proportional to their phase difference. The error signal is then low-pass filtered and used to drive a voltage-controlled oscillator (VCO) which creates an output frequency. The output frequency is fed through a frequency divider back to the input of the system, producing a negative feedback loop. If the output frequency drifts, the error signal will increase, driving the VCO frequency in the opposite direction so as to reduce the error. Thus the output is locked to the frequency at the other input.

\subsection{Phase frequency Detector}

A phase frequency detector (PFD), is a device which compares the phase of two input signals and provides a signal in the form of phase error. It has two inputs which correspond to two different input signals, usually one from a voltage-controlled oscillator (VCO) and other is a reference source. It has two outputs which instruct subsequent circuitry on how to adjust to lock onto the phase. A charge pump circuit is used to convert the digital signal from the phase frequency detector to analog signal, the output of which is used to control the frequency of the voltage control oscillator. To form a phase-locked loop (PLL), the phase error output of PFD is fed to a charge pump and then to loop filter which integrates the signal to get a sharper and smooth signal so that the disturbances at the input of VCO get minimized. As can be seen from the following diagram, the D flip-flop is an integral part of pfd. Hence to make the operation of PFD faster, a fast D flip-flop is required. 


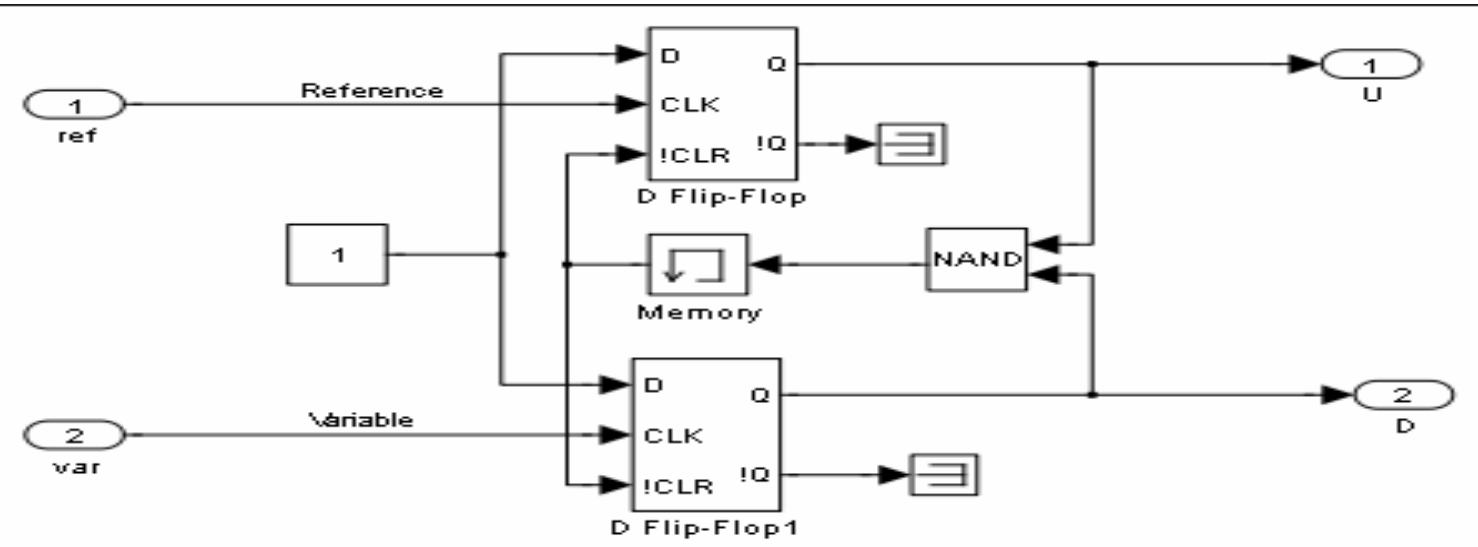

Fig. 2.1.1 Block diagram of PFD

\subsection{Frequency Divider}

Frequency divider divides the VCO frequency to generate a frequency which is comparable with reference frequency. In the above block diagram, the prescaler and swallow counter together acts as frequency divider. D flip-flop is an integral part of both of them as shown in following diagram:

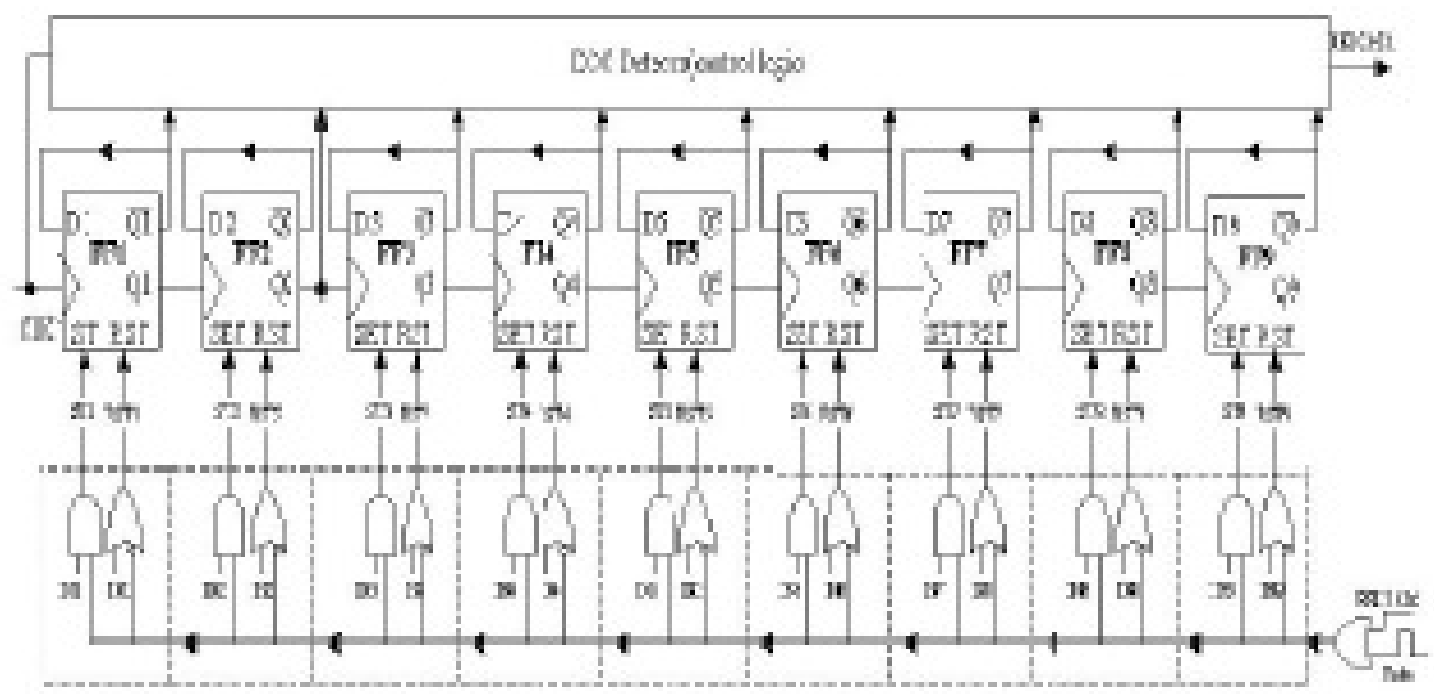

Fig. 2.2.1 Block diagram of conventional swallow counter 


\section{CONVENTIONAL D FLIP-FLOP}

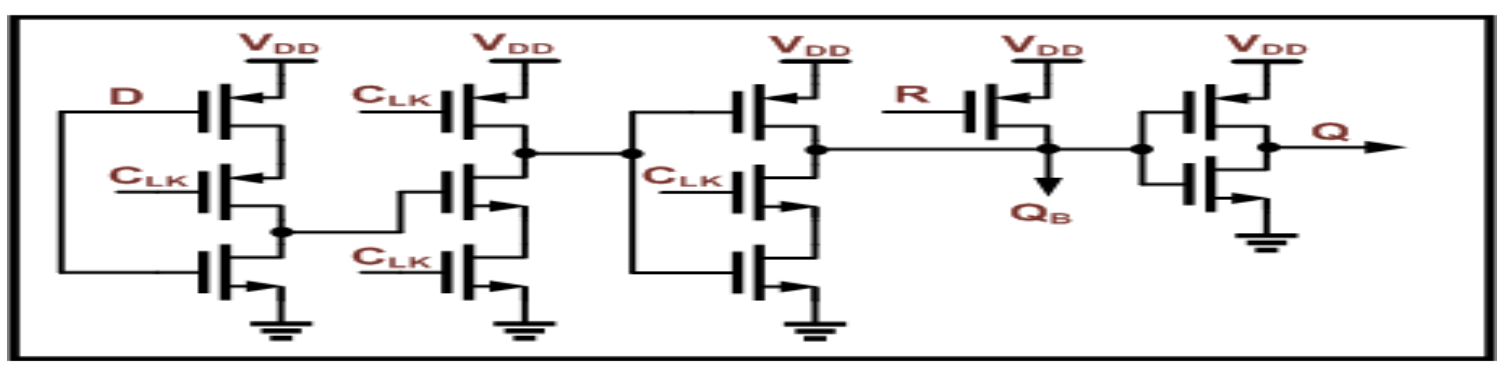

Fig 3.1 Conventional D-ff

The conventional D flip-flop which uses E-TSPC logic has higher operating frequencies but it features static power dissipation However this causes small increase in power dissipation, since at the frequencies of interest dynamic power consumption is dominant.

\section{DESIGN OF PROPOSED D FLIP-FLOP}

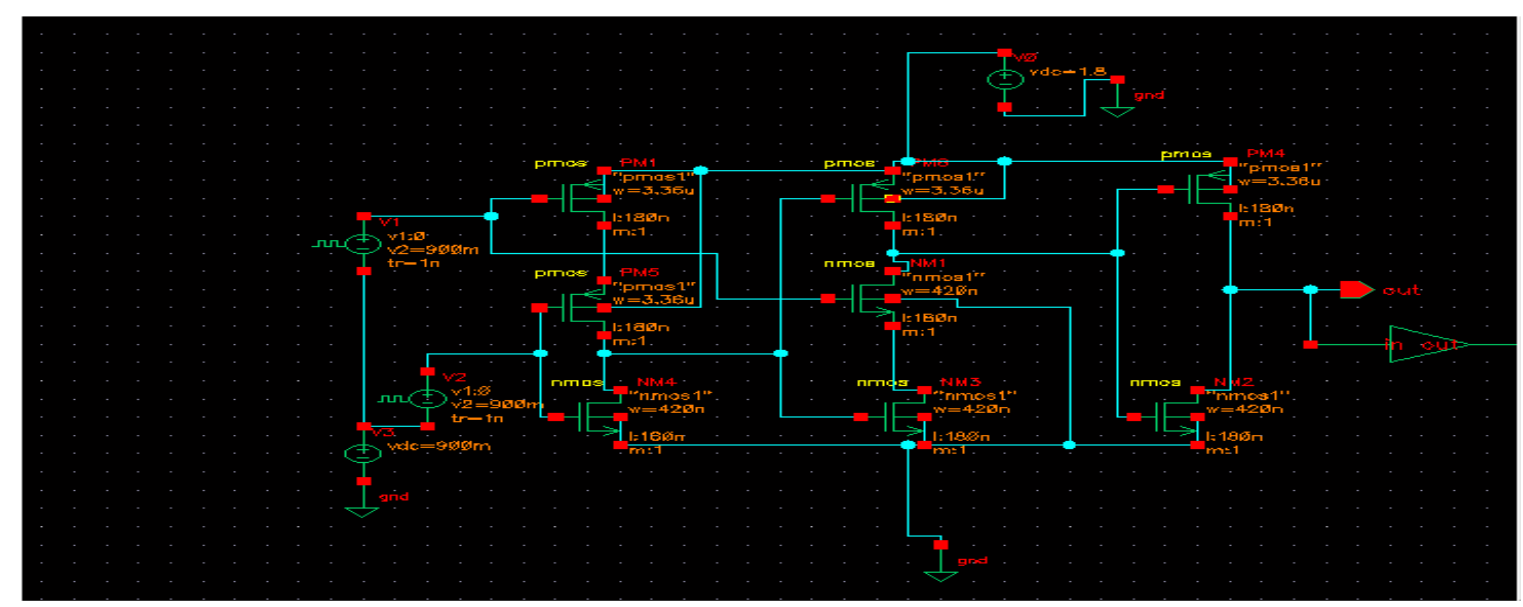

Fig .4.1 Proposed D-ff

Circuit schematic of proposed D flip-flop is as shown in figure 4.1. This flip-flop modifies the TSPC flip-flop to satisfy the required function of D-ff. When input clock (v1) and reset signal (v2) are low, a VDD is developed at the node of common drain of PM4 and NM4. At the rising edge of the clock signal (v1), a zero potential is developed at the node of common drain of PM6 and NM1. Once the node of common drain(A) of PM4 and NM4 is charged to VDD, node of common drain(B) of PM6 and NM1 is not affected by input clock signal because charges at node A turns off NM1 and this prevents the node B from being pulled up. Therefore the node B is disconnected from input node. When reset signal (v2) is applied, node A is disconnected from VDD by PM5 and is connected to ground by NM4. As soon as node A is discharged, node B is pulled up through PM6. The PM5 is added to prevent the short circuit that occurs whenever the reset signal is applied. 
International Journal of VLSI design \& Communication Systems (VLSICS) Vol.3, No.5, October 2012

\section{SIMULATION RESULTS AND WAVEFORMS}

The circuit of D-ff for phase frequency detector has been designed and simulated with $180 \mathrm{~nm}$ CMOS Technology and the result has shown in fig 5.1.The reduced power dissipation of all transistors are shown in table 5.2

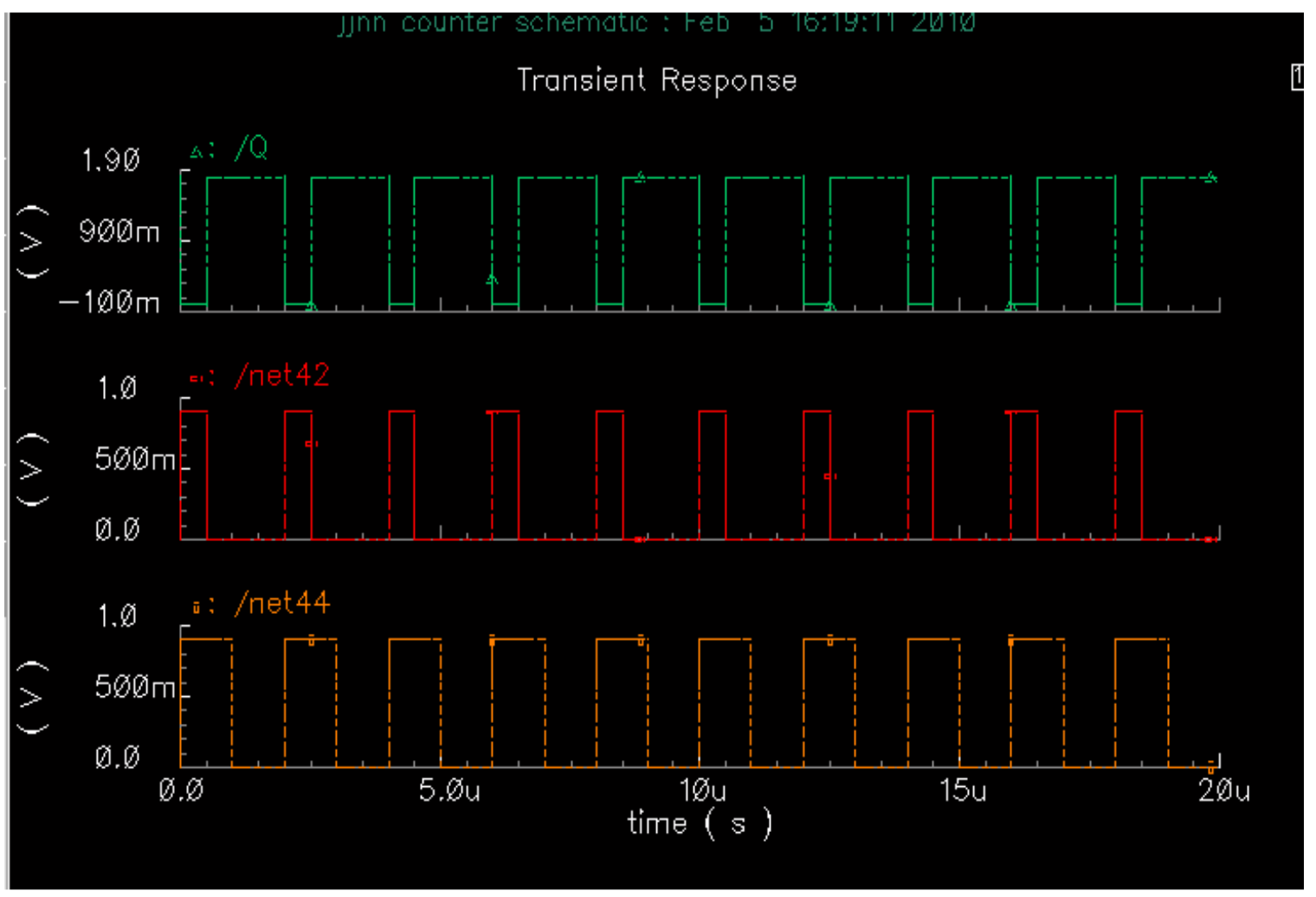

Fig .5.1 Simulation results of Proposed D-ff

Table 5.2 Simulation parameters

\begin{tabular}{|l|l|l|l|l|l|l|l|l|}
\hline Parameters & NMI & NM2 & NM3 & NM4 & PMl & PM4 & PM5 & PM6 \\
\hline Length & $180 \mathrm{nM}$ & $180 \mathrm{nM}$ & $180 \mathrm{nM}$ & $180 \mathrm{nM}$ & $180 \mathrm{nM}$ & $180 \mathrm{nM}$ & $180 \mathrm{nM}$ & $180 \mathrm{nM}$ \\
\hline Width & $0.42 \mathrm{uM}$ & $0.42 \mathrm{uM}$ & $0.42 \mathrm{uM}$ & $0.42 \mathrm{uM}$ & $3.36 \mathrm{uM}$ & $3.36 \mathrm{uM}$ & $0.46 \mathrm{uM}$ & $3.36 \mathrm{uM}$ \\
\hline Vds & $0.0448 \mathrm{~V}$ & $5.39 \mathrm{nV}$ & $1.755 \mathrm{~V}$ & $1.872 \mathrm{nV}$ & $-0.162 \mathrm{~V}$ & $-1.79 \mathrm{~V}$ & $-1.637 \mathrm{~V}$ & $-3.37 \mathrm{~V}$ \\
\hline Vth & $0.49 \mathrm{~V}$ & $0.49 \mathrm{~V}$ & $0.48 \mathrm{~V}$ & $0.49 \mathrm{~V}$ & $-0.52 \mathrm{~V}$ & $-0.51 \mathrm{~V}$ & $-0.44 \mathrm{~V}$ & $-0.52 \mathrm{~V}$ \\
\hline Pd & $2.8 \mathrm{u}$ & $2.8 \mathrm{u}$ & $2.8 \mathrm{u}$ & $2.8 \mathrm{u}$ & $7.92 \mathrm{u}$ & $7.92 \mathrm{u}$ & $7.92 \mathrm{u}$ & $7.92 \mathrm{u}$ \\
\hline Ps & $2.8 \mathrm{u}$ & $2.8 \mathrm{u}$ & $2.8 \mathrm{u}$ & $2.8 \mathrm{u}$ & $7.92 \mathrm{u}$ & $7.92 \mathrm{u}$ & $7.92 \mathrm{u}$ & $7.92 \mathrm{u}$ \\
\hline ad & $444 \mathrm{f}$ & $444 \mathrm{f}$ & $444 \mathrm{f}$ & $444 \mathrm{f}$ & $2.01 \mathrm{p}$ & $2.01 \mathrm{p}$ & $2.01 \mathrm{p}$ & $2.01 \mathrm{p}$ \\
\hline as & $444 \mathrm{f}$ & $444 \mathrm{f}$ & $444 \mathrm{f}$ & $444 \mathrm{f}$ & $2.01 \mathrm{p}$ & $2.01 \mathrm{p}$ & $2.01 \mathrm{p}$ & $2.01 \mathrm{p}$ \\
\hline
\end{tabular}


International Journal of VLSI design \& Communication Systems (VLSICS) Vol.3, No.5, October 2012

\section{CONCLUSION}

A high speed low power consumption positive edge triggered D flip-flop was presented. The D flip-flop was implemented in $180 \mathrm{~nm}$ CMOS technology. The supply voltage was $1.8 \mathrm{~V}$. In the proposed circuit dynamic power consumption was reduced by lowering internal switching and speed was increased by shortening input to output path.

\section{REFERENCES}

[1] Patrik Larsson, "High-speed Architecture for Programmable Frequency Divider and a Dual-Modulus Prescaler IEEE JOURNAL OF SOLID-STATE CIRCUITS, VOL. 31, NO. 5, MAY 1996

[2] Won-Hyo Lee, Sung-Dao Lee and Jun-Dong cho "A high speed, low power phase frequency detector and charge pump circuits for high frequency PLL", IEICE TRANS FUNDAMENTAL, vol E82-A,NO.11 November 1999

[3] Behzad Razavi, "Design of analog cmos integrated circuits", McGraw-Hill, 2001.

[4] Sedra Smith, "Microelectronics and integrated circuits"

[5] Ko-Chi Kuo, Feng-Ji Wu , "A 2.4 GHz/5-GHz Low Power Pulse Swallow Counter in 0.18 $\mu$ m CMOS technology".1-4244-0387-1/06/\$20.00@2006 IEEE

[6] Nesreen Ismail, "A Simple CMOS PFD for High Speed Applications”, European Journal of Scientific Research ISSN 1450-216X Vol.33 No.2 (2009), pp.261-269 @) EuroJournals Publishing, Inc. 2009 http://www.eurojournals.com/ejsr.htm

[7] Zheng Yong Zheng,"A 3.96 GHz phase-locked loop for mode 1 MB-OFDM UWB hopping carrier generation"Vol. 30, No. 7 Journal of Semiconductors July 2009

[8] C.C. Tien, T.M. Tien, "An 802.11a Pulse-Swallow Integer-N Frequency Synthesizer", Progress in electromagnetic Research C. Vol.7.25-35. 2009

[9] Jung-Yu Chang, Che-Wei Fan, and Shen-Iuan Liu, "A Frequency Synthesizer for Mode-1 MBOFDM UWB applications", 978-1-4244-2782-6/09/\$25.00 @2009 IEEE

[10] Chun-Lung Hsu and Wu-Hung Lu, "Glitch-Free Single-phase D-FFs for Dual-Modulus Prescrler", 07803-7889-X/03/S17.00@2003 IEEE.

\section{AUTHORS}

Prof. R. H Talwekar received the BE, ME degree in Electronics Engg.from Nagpur and Pune University in 1993 and 1997 respectively.He is currently a doctoral fellow at Nagpur University. Currently he is Associate professor of Electronics and Telecommunication Deptt. at Disha Institute of Management and Technology in Chhattisgarh Swami Vivekananda Technical University, Bhilai, Chhattisgarh India. His research interest fields are CMOS based analog mixed signal Design.

Prof (Dr). S.S. Limaye received the BE and ME in electronics engineering from VNIT, Nagpur and IIT Kharagpur, India in 1971 and 1973 respectively. He received his $\mathrm{PhD}$ degree in Electronics Engineering from Nagpur University in 1997. He worked for 15 years in industries and have been working in the field of teaching from last 21 years. Currently he is Principal at Jhulelal Institute of Technology in Nagpur University, Nagpur. His research interest fields s are FPGA based system, and CMOS based wireless IC Design.
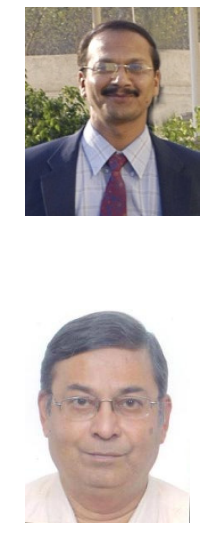